\title{
Two-Level Systems and Boson Peak Remain Stable in 110-Million-Year-Old Amber Glass
}

\author{
Tomás Pérez-Castañeda, ${ }^{1}$ Rafael J. Jiménez-Riobóo, ${ }^{2}$ and Miguel A. Ramos ${ }^{1, *}$ \\ ${ }^{1}$ Laboratorio de Bajas Temperaturas, Departamento de Física de la Materia Condensada, Condensed Matter Physics \\ Center (IFIMAC) and Instituto Nicolás Cabrera, Universidad Autónoma de Madrid, E-28049 Madrid, Spain \\ ${ }^{2}$ Instituto de Ciencia de Materiales de Madrid, Consejo Superior de Investigaciones \\ Científicas (ICMM-CSIC), E-28049 Madrid, Spain \\ (Received 7 February 2014; published 22 April 2014)
}

\begin{abstract}
The two most prominent and ubiquitous features of glasses at low temperatures, namely the presence of tunneling two-level systems and the so-called boson peak in the reduced vibrational density of states, are shown to persist essentially unchanged in highly stabilized glasses, contrary to what was usually envisaged. Specifically, we have measured the specific heat of 110 million-year-old amber samples from El Soplao (Spain), both at very low temperatures and around the glass transition $T_{g}$. In particular, the amount of two-level systems, assessed at the lowest temperatures, was surprisingly found to be exactly the same for the pristine hyperaged amber as for the, subsequently, partially and fully rejuvenated samples.
\end{abstract}

PACS numbers: $65.60 .+\mathrm{a}$, 63.50.Lm, 64.70.P-, 81.40.Cd

Amber has been appreciated by mankind for its color and natural beauty since the Neolithic era. It also has a wellknown paleontological significance, being a unique preservational system where ancient bioinclusions of animal and plant materials were stuck in the viscous resin and then fossilized for million years [1,2]. Specifically, amber is a fossilized tree resin produced from the exudates of conifers or angiosperms which has undergone a maturation process over geological time, where progressive polymerization takes place as well as evaporation of volatile components, isomerization reactions, cross-linking, and cyclization. As a result of all this, resins have been able to fossilize, after long periods of time that can exceed one hundred million years, into extremely stable materials: amber glasses. Several amber bearing deposits around the world with different types or chemical compositions of amber have proved invaluable for paleontology in the reconstruction of ecosystems and prehistoric life [1,2].

For physics and chemistry research, amber is a unique example of a glass that has been aging for a very long time below its glass transition temperature, thus reaching a state which is not accessible under normal experimental conditions. From a chemical point of view, amber is a macromolecular solid resulting of free radical polymerization. From a physical point of view, it is an amorphous solid or glass which has experienced an extreme thermodynamic stabilization process (hyperaging). As a matter of fact, amber, as many other natural or synthetic polymers, is a glass produced by chemical vitrification [3], whereas standard chemically stable glass-forming liquids become glasses by physical vitrification, i.e., by reducing temperature or increasing pressure. Nevertheless, both kinds of glass have been shown to exhibit essentially the same kinetic and thermodynamic properties characterizing the glassy behavior $[3,4]$.
Our understanding of the particularly rich phenomenology of glasses and supercooled liquids continues to be a major unsolved scientific challenge [5-10]. Whether the glass transition itself is only a purely kinetic event or the manifestation of an underlying thermodynamic transition (occurring, for instance, at the Kauzmann [11] temperature $T_{K}$, where the extrapolated entropy of the glass-forming liquid would equal that of the crystal state, if much more slowly cooled) is still under debate [6,7]. A very useful framework for interpreting the complex phenomenology of glasses and supercooled liquids is provided [12] by the potential-energy landscape (PEL). As depicted in Fig. 1, the PEL is a topographic view of the $(3 N+1)$ potentialenergy hypersurface of any glass-forming substance of $N$ particles, schematically projected on two dimensions for convenience, which has many local minima and saddle points for thermal energies below that of the melting point for the stable crystalline state (absolute minimum).

Thus, amber provides a unique benchmark to study the properties of a glass very close to its "ideal-glass" limit (as if it could be brought in thermodynamic metastable equilibrium down to $T_{K}$ ), to be followed by a comparison with the canonical glass obtained by subsequently erasing the thermal history of the amber glass (rejuvenation). One could investigate, for example, which characteristic properties of glasses are robust and inherent to the noncrystalline state, and which others are dependent on the degree of frozen-in disorder at the glass transition - and so might disappear on aging.

Specifically, our work has been aimed at studying the universal anomalous properties exhibited by glasses at low temperatures [8-10], using amber as a model system. In brief, glasses and other amorphous solids systematically exhibit at low temperature a specific heat $C_{p}$ much larger and a thermal conductivity $\kappa$ orders of magnitude lower, 


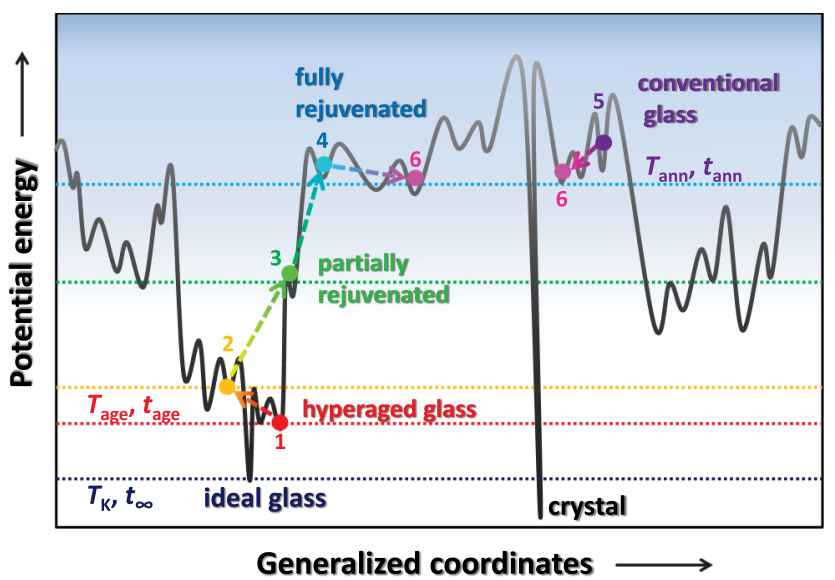

FIG. 1 (color online). Schematic potential energy landscape (PEL) for supercooled liquids and glasses, including the hypothetical absolute minimum for a crystal state. An ideal glass would be obtained after an infinitely long aging at the Kauzmann temperature $T_{K}$. The likely level for the studied hyperaging process is indicated by $T_{\text {age }}, t_{\text {age }}$. The successive states obtained with the gradual isothermal annealings (rejuvenation) at some $T_{\text {ann }}, t_{\text {ann }}$ applied to the pristine sample of amber, are illustrated with the path described by the circles and the arrows. The locations labeled 1-6 in this PEL serve to identify the different samples throughout the Letter.

respectively, than the corresponding values found in their crystalline counterparts [13]. Furthermore, $C_{p}$ was found $[8,13]$ to depend quasilinearly $\left(C_{p} \propto T^{1+\delta}\right)$ and $\kappa$ almost quadratically $\left(\kappa \propto T^{2-\delta}\right.$ ) on temperature $T$, in clear contrast to the cubic dependences observed in crystals for both properties, well understood in terms of Debye's theory of lattice vibrations. These and related acoustic and dielectric properties of amorphous solids at low temperatures [8] were soon well accounted for [14,15] by the successful tunneling model (TM), though some open questions remain unsolved [16].

On the other hand, the thermal behavior of glasses above $1 \mathrm{~K}$ and their corresponding low-frequency vibrational properties around $1 \mathrm{THz}$, are much more poorly understood. This frequency range is indeed dominated by another universal and much disputed feature of glasses: the so-called "boson peak" $[8,10]$ arising from a noteworthy excess in the vibrational density of states (VDOS) over that predicted by Debye's theory $g(\omega) \propto \omega^{2}$. Such an excess in the lowfrequency VDOS appears as a broad peak in $g(\omega) / \omega^{2}$, which produces the broad maximum in $C_{p} / T^{3}$ observed in most glasses at a few $\mathrm{K}$.

In this Letter, we have measured the specific heat of several amber samples from El Soplao, in the low-temperature range $0.07 \mathrm{~K}<T<30 \mathrm{~K}$, through their gradual rejuvenation and corresponding characterization, including elasto-optic measurements below room temperature and temperaturemodulated differential scanning calorimetry (TM-DSC) around the glass transition. The details about the experimental methods and calculations are included in the Supplemental Material [17].

Specific-heat curves around the glass transition in different states of amber, ranging from a pristine sample to one quenched from the liquid, are plotted in Fig. 2(a). Several different isothermal annealing processes were applied to pristine amber samples, trying to follow the route depicted in the PEL of Fig. 1. As expected, these annealing treatments below $T_{g}$ destabilize and increase the internal energy of the previously hyperaged glass, oppositely to usual annealing processes for conventional glasses. In Fig. 2(b), we present the corresponding enthalpy curves, obtained by integration of the former curves through
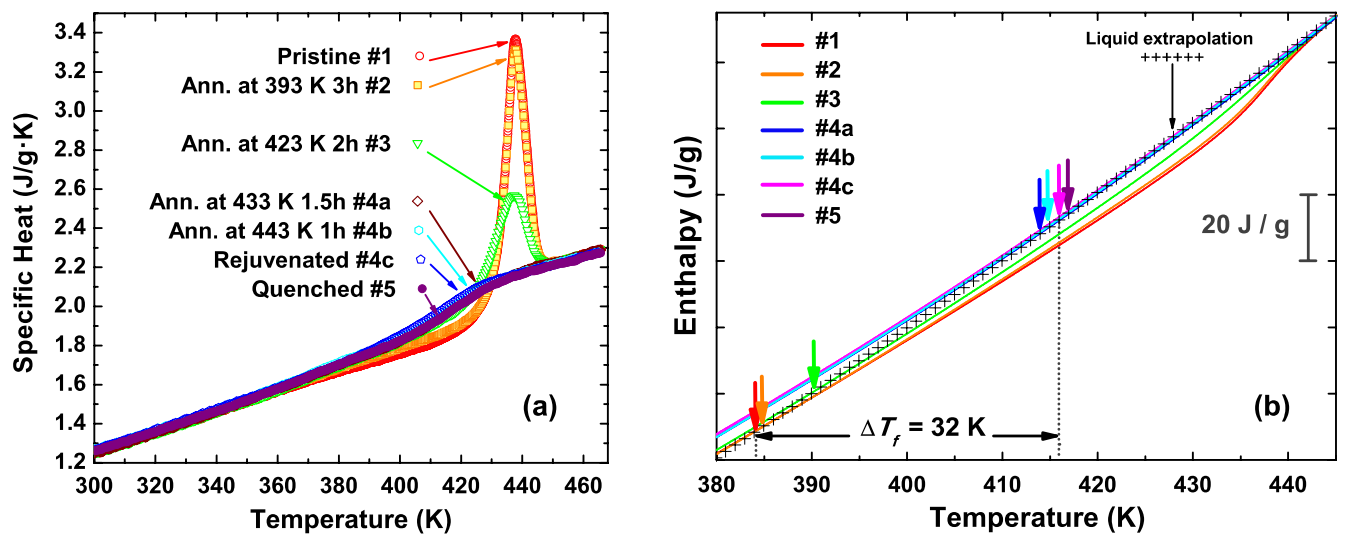

FIG. 2 (color online). (a) Specific heat of amber from El Soplao at different states, measured by TM-DSC with heating rates of $1 \mathrm{~K} / \mathrm{min}$ and modulating amplitude $\pm 0.5 \mathrm{~K} / \mathrm{min}$ every $80 \mathrm{~s}$. Only the first upscan (see the Supplemental Material [17]) for each sample is presented. The devitrification temperature of the pristine sample is located at $T_{g}^{*}=438 \mathrm{~K}$, and then decreases with decreasing stability (rejuvenation). The aging signal is clearly seen as a huge endothermic peak at the glass transition, which is maximal in the case of the pristine sample 1. Rejuvenation of amber was done stepwise (Table I) by performing different isothermal treatments to the pristine sample near the glass transition. (b) Corresponding enthalpy curves from the specific-heat ones in (a), running from lower to upper solid curves when going from sample 1 to sample 5 in the same order as in the legend. The fictive temperature $T_{f}$ for each sample (indicated by the arrows, going from left to right following the same order as in the legend) is obtained as the intersection of the extrapolated liquid curve $(+)$ and the glass curve extrapolated from temperatures well below its $T_{g}^{*}$ (see the Supplemental Material [17]). 


$$
\Delta H=\int_{T}^{T_{0}} C_{P}(T) d T,
$$

with $T_{0}$ being a reference temperature in the liquid state.

As can be seen in Fig. 2(a), a huge endothermic peak is observed for the pristine amber (sample 1) at the calorimetric devitrification temperature $T_{g}^{*}=438 \mathrm{~K}$ [determined by the inflection point of the reversing $C_{p}$ jump (see the Supplemental Material [17])]. $T_{g}^{*}$ is well above the genuine glass transition temperature $T_{g}=423 \mathrm{~K}$ obtained for the rejuvenated sample $4 \mathrm{c}$, or alternatively from the second or third heating runs for any sample [17], when the cooling and heating rates are canonically the same. This unusual increase of the calorimetric $T_{g}^{*}$ for the stabilized amber compared to the canonical glass, has been ascribed to a high kinetic stability in the related case of ultrastable thin films of organic glasses [23], indicating that much higher temperatures are needed to dislodge the molecules from their glassy configurations.

The fictive temperature $T_{f}$ is defined [24] as the temperature at which the nonequilibrium (glass) state and its equilibrium (supercooled liquid) state would have the same structure and properties, in particular, enthalpy. As depicted in Fig. 2(b), we have determined $T_{f}$ as the intersection point (marked by the arrows) between the extrapolated enthalpy of the liquid and that of the corresponding glass at temperatures well below its $T_{g}^{*}$. Obtained data are given in Table I. The observed extraordinary decrease $\Delta T_{f}=32 \mathrm{~K}$ (thermodynamic stability) for the pristine amber compared to the rejuvenated glass is similar or even superior to the effects seen in some ultrastable thin films of organic glasses $[23,25,26]$. Such a reduction over $9 \%$ of the fictive temperature with respect to the substance glass transition is, thus, the consequence of extremely prolonged sub-sub- $T_{g}$ structural relaxations [27]. Notice that $T_{g}^{*} \neq T_{f}$ even for the canonical rejuvenated glass, what is mainly due to the specific calorimetric method employed to determine $T_{g}^{*}$ (see the Supplemental Material [17]).

Following the theoretical route upwards through the PEL (Fig. 1), we experimentally see (Fig. 2) that applying an isothermal annealing for three hours at $393 \mathrm{~K}$ (sample 2), well below the glass transition $T_{g}=423 \mathrm{~K}$, only produces a very slight change in the pristine sample. When we further increase the annealing temperature, approaching it to $T_{g}$, both the endothermic $C_{p}$ peak and the enthalpy variation gradually decrease (sample 3, partial rejuvenation) until they completely disappear already for the thermal annealing at $433 \mathrm{~K}$ (sample 4a). Calorimetric curves after isothermal annealings (samples 4a and 4b) are indistinguishable from that for the fully rejuvenated sample (sample 4c), obtained after heating it up to $470 \mathrm{~K}$, well in the liquid state. Moreover, quenching the liquid at $50 \mathrm{~K} / \mathrm{min}$ (sample 5) makes no significant difference from the conventional glass in the specific-heat and enthalpy curves. We have also tried to restabilize a rejuvenated amber glass, by annealing it for two hours at
TABLE I. Calorimetric (devitrification) glass-transition temperatures $T_{g}^{*}$ and fictive temperatures $T_{f}$ obtained after the different thermal histories applied to the studied samples. The expected location in the potential-energy landscape (PEL) of Fig. 1 is indicated in the second column and serves as a label for the samples. The last column displays the relative decrease of the fictive temperature $T_{f}$ in relation to the standard glass-transition temperature of this Spanish amber, $T_{g}=423 \mathrm{~K}$.

\begin{tabular}{lcccc}
\hline \hline & $\begin{array}{c}\text { PEL sample } \\
\text { number }\end{array}$ & $\begin{array}{c}T_{g}^{*} \\
(\mathrm{~K})\end{array}$ & $\begin{array}{c}T_{f} \\
(\mathrm{~K})\end{array}$ & $\frac{T_{f}-T_{g}}{T_{g}}$ \\
\hline Pristine (hyperaged) & 1 & 438 & 384 & $-9.2 \%$ \\
$3 \mathrm{~h}$ at $393 \mathrm{~K}$ & 2 & 438 & 385 & $-9.0 \%$ \\
$2 \mathrm{~h}$ at $423 \mathrm{~K}$ & 3 & 436 & $391-7.6 \%$ \\
$1.5 \mathrm{~h}$ at $433 \mathrm{~K}$ & $4 \mathrm{a}$ & 423 & 413 & $-2.4 \%$ \\
$1 \mathrm{~h}$ at $443 \mathrm{~K}$ & $4 \mathrm{~b}$ & 423 & $415-1.9 \%$ \\
Rejuvenated $(>460 \mathrm{~K})$ & $4 \mathrm{c}$ & 423 & $416-1.7 \%$ \\
Quenched & 5 & 423 & $417-1.4 \%$ \\
Rejuvenated and annealed & 6 & 424 & $416-1.7 \%$ \\
$\quad 2 \mathrm{~h}$ at $423 \mathrm{~K}$ & & & & \\
\hline \hline
\end{tabular}

$423 \mathrm{~K}$ (sample 6), but it again produced almost negligible effects on both the thermodynamic and kinetic stability of the glass (Table I).

Finally, our main aim was to study the influence of the above-confirmed dramatic stabilization of the hyperaged glass on its low-temperature properties. We present in Fig. 3 our specific-heat measurements for pristine amber (sample 1), a partially rejuvenated sample (sample 3), and the fully rejuvenated one (sample 4c). Figure 3(a) is a log-log plot at the lowest temperatures, which makes more clearly visible that the two-level-systems (TLS)-dominated lowtemperature specific heat remains invariable within experimental error. This is the main result of our Letter. On the other hand, above $1 \mathrm{~K}$, the specific heat moderately increases with rejuvenation around the boson peak in $C_{p} / T^{3}$ [occurring at $3.4 \pm 0.1 \mathrm{~K}$ in all cases, see Fig. 3(b)], following the same trend as the elastic Debye coefficient obtained from Brillouinscattering and mass-density measurements (see Table II and the Supplemental Material [17]). As can be seen there, the aging process in amber has produced a densification of around $2 \%$. The combination of mass-density and sound-velocity variations translates into a 9\% lower Debye coefficient for the hyperaged (pristine) amber than for the rejuvenated (canonical) glass.

A strong $C_{p} / T^{3}$ peak was already observed by us [28] in pristine (20 million years old) Dominican amber, though apparent residual curing or repolymerization, occurring around the glass transition temperature when rejuvenating those amber samples, hindered a reliable quantitative investigation. Also stress-relaxation experiments in glass and supercooled-liquid states of Dominican amber have been recently reported [29].

Whether or not the low-temperature universal "anomalies" of glasses (i.e., TLS and boson peak) could be eventually suppressed by much stronger and longer annealing or aging processes, and hence, whether they 

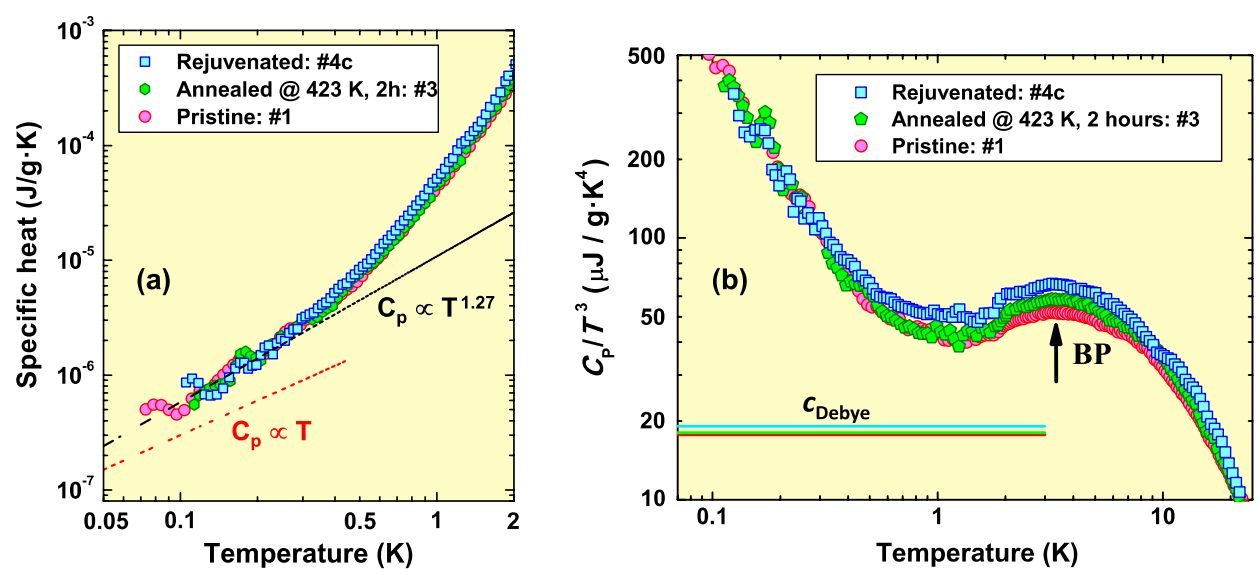

FIG. 3 (color online). (a) Comparison of specific-heat data for three Spanish amber samples (pristine, partially rejuvenated, and fully rejuvenated) at very low temperatures, 0.05-2 K. The upper dashed line shows the best quasilinear fit to the experimental data below $0.4 \mathrm{~K}$ given by $C_{p} \propto T^{1.27}$, hence faster than the simple linear dependence indicated by the lower dashed line. (b) $C_{p} / T^{3}$ plot of the same data in (a), displayed in a wider temperature range. The height of the boson peak is observed to further increase with rejuvenation, though the values of both the minimum and the maximum of $C_{p} / T^{3}$ remain constant: $T_{\min }=1.2 \pm 0.1 \mathrm{~K}$ and $T_{\max }=3.4 \pm 0.1 \mathrm{~K}$, respectively. The corresponding Debye levels determined from the sound velocity and density data (Table II) are indicated by solid lines and exhibit the same trend as the boson-peak height (both sets follow the same order as the symbols in the legend).

are or not intrinsic properties of the glass state, has been a long-standing question. During the last forty years, different experiments [30-36] have been reported about the possible influence of the thermal history on these properties, with contradictory conclusions. Many of them were focused on the paradigmatic vitreous silica. In brief, a modest decrease of the low-temperature specific heat with annealing was usually found [31], both at the lowest temperatures and around the $C_{p} / T^{3}$ maximum. However, the important role played by the different amounts of water content, as well as the lack of measured elasto-optic data with thermal treatment and hence, of its variable contribution to the total heat capacity, makes their conclusions unclear. The same applies to early low-temperature specific-heat measurements in glassy glycerol [30], where the height of the $C_{p} / T^{3}$ maximum at $9 \mathrm{~K}$ was found to be a $7 \%$ lower in a slowly cooled glass than in a quenched glass. Also, a decrease with annealing of both the $C_{p} / T^{3}$ maximum and the $g(\omega) / \omega^{2}$ boson peak inferred from Raman scattering was reported in $\mathrm{As}_{2} \mathrm{~S}_{3}$ glasses [32]. Nonetheless, later Raman and inelastic neutron scattering experiments [33] in the same glasses suggested that the observed changes in the total VDOS were caused by changes of sound velocity and density as a result of quenching. A similar conclusion was reached in dry
$\mathrm{B}_{2} \mathrm{O}_{3}$ glasses [34], where very different $C_{p} / T^{3}$ peaks for different thermal treatments were found to merge into a single curve after their corresponding Debye levels had been subtracted. Interestingly, careful inelastic neutron scattering experiments in polybutadiene [35] showed a clear absence of annealing effect in its VDOS (boson peak), though a slight decrease in the boson peak was reported [36] after physical aging in another polymer, PMMA.

Indeed, some authors have tried to correlate the boson peak feature in glasses [37], and even in crystals [38], with transformations of the elastic continuum only. In the case of amber, however, such a Debye-scaling rule does not hold quantitatively. The height of the $C_{p} / T^{3}$ boson peak in the hyperaged amber has decreased $22 \%$ from the standard rejuvenated glass, whereas a Debye scaling [37] $\left(\propto \omega_{D}{ }^{-3}\right.$, with $\omega_{D}$ being the Debye frequency) would predict only a $7.4 \%$ reduction. A similar relation was proposed by Shintani and Tanaka [39]. From numerical simulations in 2D glassforming systems, they suggested that the boson peak height should scale with the inverse of the shear modulus. From the data in Table II, one would expect a boson-peak reduction $<7 \%$, again well below the experimentally observed $22 \%$.

Our experiments on hyperaged glasses of amber, far away from laboratory time scale annealing or quenching processes, undoubtedly demonstrate that these ideal-like glasses,

TABLE II. Measured mass density at room temperature $\rho_{\mathrm{RT}}$ and zero-temperature extrapolated $\rho(0)$, longitudinal $\mathrm{v}_{L}(0)$, and transverse $\mathrm{v}_{T}(0)$ sound velocity, average Debye velocity in the zero-temperature limit $\mathrm{v}_{D}$, calculated cubic Debye coefficient $c_{D}$ for the specific heat (see the Supplemental Material [17]) and height of the $C_{p} / T^{3}$ boson peak.

\begin{tabular}{|c|c|c|c|c|c|c|c|}
\hline Sample & $\rho_{\mathrm{RT}}\left(\mathrm{kg} / \mathrm{m}^{3}\right)$ & $\rho(0)\left(\mathrm{kg} / \mathrm{m}^{3}\right)$ & $\mathrm{v}_{L}(0)(\mathrm{m} / \mathrm{s})$ & $\mathrm{v}_{T}(0)(\mathrm{m} / \mathrm{s})$ & $\mathrm{v}_{D}(\mathrm{~m} / \mathrm{s})$ & $\mathrm{c}_{D}\left(\mu \mathrm{Jg}^{-1} \mathrm{~K}^{-4}\right)$ & $\left(C_{P} / T^{3}\right)_{\mathrm{BP}}\left(\mu \mathrm{Jg}^{-1} \mathrm{~K}^{-4}\right)$ \\
\hline Pristine sample 1 & 1045 & 1055 & 3175 & 1635 & 1831 & 18.9 & 51.9 \\
\hline Annealed sample 3 & 1038 & 1049 & 3160 & 1625 & 1820 & 19.3 & 58.1 \\
\hline Rejuvenated sample $4 \mathrm{c}$ & 1024 & 1035 & 3115 & 1596 & 1788 & 20.7 & 66.4 \\
\hline
\end{tabular}


subjected to a dramatic thermodynamic stabilization, do exhibit the same low-temperature properties of the conventional (rejuvenated) glass. Erasing the strong structural relaxation and enthalpy reduction of pristine amber only produces a modest quantitative increase in the height of the $C_{p} / T^{3}$ peak that could be qualitatively ascribed to the corresponding variation of the elastic constants and the Debye coefficient. Furthermore, the best fingerprint of the universal glassy anomalies is surely the density of TLS, measured from the corresponding quasilinear contribution to the specific heat, $C_{p} \propto T^{1+\delta}$, since the influence of Debye-like lattice vibrations becomes less and less important below $1 \mathrm{~K}$. In this respect, our experimental results are conclusive: pristine, partially rejuvenated and fully rejuvenated amber glasses have the same specific heat below $1 \mathrm{~K}$, within experimental error.

The boson peak and the tunneling TLS are, therefore, robust and intrinsic properties of glasses which remain "fossilized" in 110-million-year stabilized glasses of amber, as insects or other bioinclusions do. We also expect that amber, a hyperaged glass, will work as an extremely enlightening model glass to study many other puzzles involved in the physics of the glass state.

This work was financially supported by the Spanish MINECO (Projects No. FIS2011-23488 and No. MAT2012-37276-C03-01) and partially by the Autonomous Community of Madrid (Project No. PHAMA S2009/MAT-1756). T. P. C. acknowledges financial support from the Spanish Ministry of Education through the FPU Grant No. AP2008-00030. The authors are especially grateful to Idoia Rosales and César Menor-Salván for providing the El Soplao amber samples, to Tomás E. Gómez for his collaboration with ultrasonic experiments, and to Uli Buchenau for helpful discussions and a critical reading of the Letter. María José de la Mata is gratefully acknowledged for her technical support with TM-DSC measurements conducted at SIdI-UAM. Pilar Miranzo is thanked for her help with mass-density measurements.

*miguel.ramos@uam.es

[1] J. B. Lambert and G. O. Poinar, Jr., Acc. Chem. Res. 35, 628 (2002), and references therein

[2] D. Grimaldi, Science 326, 51 (2009), and references therein.

[3] S. Corezzi, D. Fioretto, and P. Rolla, Nature (London) 420 , 653 (2002).

[4] S. Caponi, S. Corezzi, D. Fioretto, A. Fontana, G. Monaco, and F. Rossi, Phys. Rev. Lett. 102, 027402 (2009).

[5] C. A. Angell, K. L. Ngai, G. B. McKenna, P. F. McMillan, and S. W. Martin, J. Appl. Phys. 88, 3113 (2000).

[6] P. G. Debenedetti and F. H. Stillinger, Nature (London) 410, 259 (2001).

[7] A. Cavagna, Phys. Rep. 476, 51 (2009).

[8] W. A. Phillips, Amorphous Solids: Low-Temperature Properties (Springer, Berlin, 1981).

[9] P. Esquinazi, Tunneling Systems in Amorphous and Crystalline Solids (Springer, Berlin, 1998).

[10] R. Zorn, Physics 4, 44 (2011).
[11] W. Kauzmann, Chem. Rev. 43, 219 (1948).

[12] M. Goldstein, J. Chem. Phys. 51, 3728 (1969).

[13] R. C. Zeller and R. O. Pohl, Phys. Rev. B 4, 2029 (1971).

[14] W. A. Phillips, J. Low Temp. Phys. 7, 351 (1972).

[15] P. W. Anderson, B. I. Halperin, and C. M. Varma, Philos. Mag. 25, 1 (1972).

[16] C. C. Yu and A. J. Leggett, Comments Condens. Matter Phys. 14, 231 (1988).

[17] SeeSupplemental Material athttp://link.aps.org/supplemental/ 10.1103/PhysRevLett.112.165901 for the description of the samples, their calorimetric characterization through TM-DSC, the method followed to determine the fictive temperatures, the experimental methods and detailed calculations of the zerotemperature extrapolations for the elasto-optic coefficients, and experimental details on low-temperature heat capacity measurements.

[18] C. Menor-Salván, M. Najarro, F. Velasco, I. Rosales, F. Tornos, and B. R. T. Simoneit, Organic Geochemistry 41, 1089 (2010).

[19] M. Najarro, E. Peñalver, I. Rosales, R. Pérez-de la Fuente, V. Daviero-Gómez, B. Gómez, and X. Delclós, Geologica Acta 7, 363 (2009).

[20] J. K. Krüger, J. Baller, T. Britz, A. le Coutre, R. Peter, R. Bactavatchalou, and J. Schreiber, Phys. Rev. B 66, 012206 (2002).

[21] T. Pérez-Castañeda, J. Azpeitia, J. Hanko, A. Fente, H. Suderow, and M. A. Ramos, J. Low Temp. Phys. 173, 4 (2013).

[22] E. Pérez-Enciso and M. A. Ramos, Thermochim. Acta 461, 50 (2007).

[23] S. F. Swallen, K. L. Kearns, M. K. Mapes, Y. S. Kim, R. J. McMahon, M. D. Ediger, T. Wu, L. Yu, and S. Satija, Science 315, 353 (2007).

[24] A. Q. Tool, J. Am. Ceram. Soc. 29, 240 (1946).

[25] K. L. Kearns, S. F. Swallen, M. D. Ediger, T. Wu, Y. Sun, and L. Yu, J. Phys. Chem. B 112, 4934 (2008).

[26] A. Sepúlveda, E. León-Gutiérrez, M. González-Silveira, C. Rodríguez-Tinoco, M. T. Clavaguera-Mora, and J. Rodríguez-Viejo, Phys. Rev. Lett. 107, 025901 (2011).

[27] S. Brawer, Relaxation in Viscous Liquids and Glasses (American Ceramic Society, Columbus, OH, 1985).

[28] T. Pérez-Castañeda, R. J. Jiménez-Riobóo, and M. A. Ramos, J. Phys. Condens. Matter 25, 295402 (2013).

[29] J. Zhao, S. L. Simon, and G. B. McKenna, Nat. Commun. 4, 1783 (2013).

[30] R. Calemczuk, R. Lagnier, and E. Bonjour, J. Non-Cryst. Solids 34, 149 (1979).

[31] H. v. Löhneysen, H. Rüsing, and W. Sander, Z. Phys. B 60, 323 (1985).

[32] N. Ahmad, K. Hutt, and W. A. Phillips, J. Phys. C 19, 3765 (1986).

[33] S. L. Isakov, S. N. Ishmaev, V. K. Malinovsky, V. N. Novikov, P. P. Parshin, S. N. Popov, A. P. Sokolov, and M. G. Zemlyanov, Physica (Amsterdam) 201A, 386 (1993).

[34] E. Pérez-Enciso, M. A. Ramos, and S. Vieira, Phys. Rev. B 56, 32 (1997).

[35] R. Zorn and B. Frick, J. Chem. Phys. 108, 3327 (1998).

[36] E. Duval, L. Saviot, L. David, S. Etienne, and J. F. Jal, Europhys. Lett. 63, 778 (2003).

[37] A. Monaco, A. I. Chumakov, Y.-Z. Yue, G. Monaco, L. Comez, D. Fioretto, W. A. Crichton, and R. Rüffer, Phys. Rev. Lett. 96, 205502 (2006).

[38] A. I. Chumakov et al., Phys. Rev. Lett. 112, 025502 (2014).

[39] H. Shintani and Y. Tanaka, Nat. Mater. 7, 870 (2008). 